\title{
Bletilla striata Polysaccharides: Structures and Functional Activities
}

\author{
Jinyu Yi, ${ }^{a}$ Yingying Huang, ${ }^{a}$ and Chaoyan Zhang ${ }^{*, a, b, c, d}$ \\ ${ }^{a}$ College of Food Science and Technology, Shanghai Ocean University, Shanghai 201306, China \\ ${ }^{b}$ Shanghai Engineering Research Center of Aquatic-Product Processing \& Preservation, Shanghai 201306, \\ China \\ ${ }^{c}$ Laboratory of Quality and Safety Risk Assessment for Aquatic Product on Storage and Preservation \\ (Shanghai), Ministry of Agriculture, Shanghai 201306, China \\ ${ }^{d}$ Collaborative Innovation Center of Seafood Deep Processing, Dalian Polytechnic University, Dalian, \\ Liaoning 116034, China \\ Email: chyzhang@shou.edu.cn (C. Z.)
}

\begin{abstract}
Bletilla striata (Thunb.) Reichb.f. is a species of genus Bletilla (Family Orchidaceae) and it is a commonly used traditional Chinese medicine. Bletilla striata polysaccharides (BSPs) were extracted from the tuber of Bletilla striata. Herein, we reviewed the extraction and purification methods of BSPs, compared the advantages and disadvantages of different methods comprehensively, and analyzed molecular weights and structural characterization of BSPs systematically. In addition, we analyzed hemostasis, anti-oxidation, anti-aging and other functional activities of BSPs to provide scientific basis for further study of BSPs.
\end{abstract}

Keywords Bletilla striata polysaccharides, structures, functional activities

\section{Introduction}

Bletilla striata (Thunb.) Reichb.f. is a kind of Chinese traditional medicine widely distributed in East Asia. Its dried tubers are widely used as a hemostatic agent. ${ }^{[1]}$ It is reported that these polysaccharides are the most important biologically active components of $B$. striata with various bioactivities. ${ }^{[2]}$ Clinically, it is mainly used to treat hemoptysis, traumatic hemorrhage, gastrointestinal mucosal injury, ulcers, bruises and burns, anorectal diseases, gynecological fibroma, tumor embolization and prostate surgery, etc. ${ }^{[3-6]}$ Polysaccharides were extracted from $B$. striata. In the pharmaceutical industry, BSPs have been widely applied as biomaterials with good mechanical properties and unique biological functions. ${ }^{[7]}$ Most of BSP are water-soluble polysaccharides, which are considered as the main active ingredients with the above-mentioned medicinal functions. As a promising natural anti-oxidant or excellent moisturizer, they are widely used in cosmetics and chemical industries.

Pharmacological research on $B$. striata shows that BSPs are a glucomannan formed from glucose and mannose $(1: 4)$ polymerized with $\beta$-glycosidic bonds. ${ }^{[8]}$ As a natural polymer material, BSPs have the characteristics of functional slowrelease, local retention, self-degradability and non-irritating. It is increasingly valued in the process of pharmaceutical preparation and application ${ }^{[9]}$ with wide application prospects. ${ }^{[10,11]}$ BSPs have high safety as food additives or ingredients, and they are widely used in food processing industry due to their unique properties. ${ }^{[12]}$ Herein, we summarized extraction, purification, structural characterization and functional activities of BSPs for more reference values in future.

\section{Extraction, Separation and Purification of BSPs \\ Extraction of BSPs}

At present, the extraction methods of BSPs include traditional extraction methods and new extraction techniques.
In the traditional method, the extraction of polysaccharides mainly uses the reflux method, and the conventional method requires longer processing time and lower efficiency. The reflux temperature is not easy to control, and it is easy to consume a lot of volatiles and harmful organic solvents, causing environmental pollution. At the same time, the reflux process is likely to cause deformation and degradation of polysaccharides, resulting in the reduction of sample purity. ${ }^{[13]}$ At present, the most commonly used method to extract BSPs is water extraction and alcohol precipitation. Kong et al. ${ }^{[14]}$ reported that the dried tubers of $B$. striata were ground into powder and the ratio of material to liquid $(V / V)$ was controlled. Then, they were extracted for $4 \mathrm{~h}$ at $80{ }^{\circ} \mathrm{C}$, filtrated, adsorbed with $0.5 \%$ activated carbon at $60{ }^{\circ} \mathrm{C}$ for $2 \mathrm{~h}$, and precipitated for $2 \mathrm{~h}$ with $75 \%$ ethyl alcohol. After that, the precipitate was centrifugated, dissolved and freeze-dried. Under this condition, the extraction rate of BSPs is $28.96 \%$. Nowadays, various methods have been developed for extraction of BSPs. Table 1 shows the advantages and disadvantages of various extraction methods of BSPs.

\section{Separation and purification of BSPs}

The crude polysaccharides obtained by extraction needs to be further purified by separation and purification. Sevage is a common method for removing free protein from polysaccharides. It is relatively mild and has a little effect on the structures of polysaccharides. It can effectively avoid protein residue after repeated use. ${ }^{[22]}$ Column chromatography is widely used in the purification of BSPs due to its simple operation and good purification performance. Anion exchange chromatography is suitable for separation of various polysaccharides, while gel permeation chromatography is generally not suitable for separation of mucopolysaccharides. ${ }^{[22]}$ As a kind of mucopolysaccharide, BSPs should be loaded into anion exchange chromatography firstly, and then DEAE-cellulose exchanger was used for chromatography to obtain separated components. 
Table 1 Comparison of extraction methods of BSPs.

\begin{tabular}{|c|c|c|c|}
\hline Method & Advantage & Disadvantage & Ref. \\
\hline Hot water extraction & The most widely used; easy to operate & This method took long time & {$[17,18]$} \\
\hline Ultrasonic extraction & Widely used; no heating; high efficiency & High yield energy & {$[15-19]$} \\
\hline Infrared assisted extraction & Short extraction time; low extraction temperature & High yield energy & {$[16]$} \\
\hline Enzyme-assisted method & High extraction & $\begin{array}{l}\text { Rarely used alone, usually } \\
\text { combined with other method }\end{array}$ & [20] \\
\hline $\begin{array}{l}\text { Dilute alkaline water } \\
\text { extraction }\end{array}$ & Mild reaction conditions and high efficiency & - & [21] \\
\hline
\end{tabular}

In the subsequent purification process, each component of BSPs can be separated more carefully according to different molecular weights. With the monitoring of the phenol sulfuric acid method, the two chromatographic methods can be used interchangeably, and the product can be further purified. After concentration, the purified BSPs were obtained from eluent. ${ }^{[2]}$ Finally, purified BSPs have been widely studied in many aspects (Figure 1).

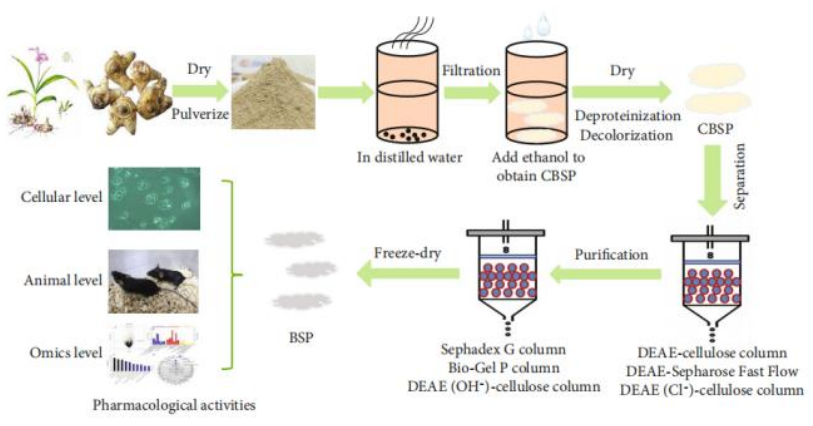

Figure 1 Schematic representation of the extraction, purification and bioactivity of BSPs. ${ }^{[2]}$

\section{Portion weights of BSPs}

BSPs are a kind of compound polymerized by many monosaccharides linked by glycoside bond. BSPs were a glucomannan consisting of 4 molecules of mannose and 1 molecule of glucose. There are also a few metal ions in the molecule. At the same time, there are three kinds of residues including $\beta$-1,4-mannose, $\beta$-1,4-glucose and $\alpha$-1,6glucose. ${ }^{[23,24]}$ The relative molecular weights of BSPs range from tens of thousands to millions. ${ }^{[25,26,28]}$ The relative molecular weights of polysaccharides only represent the average distribution of a certain range of relative molecular weights. Even if the same batch of polysaccharides, different relative molecular weights are often measured by different methods. Chen et al. ${ }^{[23]}$ used high performance liquid gel permeation chromatography (HPGPC) to measure the molecular weights of BSPs $\left(9.9658 \times 10^{4}\right)$. Lu et al. ${ }^{[27]}$ measured the average molecular weights of BSPs were 183,000 by Sephadex G-200 gel filtration method. Liu et al. ${ }^{[28]}$ used $B$. striata tuber as material, which was extracted three times at $80{ }^{\circ} \mathrm{C}$ with ultrasonic method, ethanol precipitated, removed of protein by Sevage method. Finally, two polysaccharide samples were obtained by ultrafiltration treatment and gel column (Sephadex G-200) chromatography. The relative molecular weight is 460 $\mathrm{kDa}$ and $390 \mathrm{kDa}$, respectively.

\section{Structural characterization of BSPs}

The primary structure of polysaccharide refers to the composition and sequence of monosaccharide residues, adjacent glycosyl connections, anomeric configuration, and the location and length of branches. Though the reports on the structural fractions of BSPs have been in update, all the results indicated that BSPs were a sort of glucomannan (Table 2). An early publication in 1973 reported firstly that BSPs contained a mannose to glucose in a molar ratio of $3: 1$, and its skeleton is composed of $(1 \rightarrow 4)$ linked aldonic sugar residues. ${ }^{[30]}$ In 2006 , 2010, 2014 and 2017, more new BSPs components were extracted with different molecular weights and monosaccharide compositions. ${ }^{[29,31-33]}$ The skeleton of BSPs is mainly composed of 1,4 linked mannose residues and 1,4 linked glucose residues. In addition, the monosaccharide composition of BSPs is mainly mannose and glucose, only bspf2 contains a small amount of galactose units. ${ }^{[1,29-33]}$ In 2015, it could be referred to as glucomannan polymer. ${ }^{[34]}$ In addition, there is a certain diversity in the molecular weight of each BSP component, which remains in the range of $1-3 \times 10^{5}$. The hypothesized structure of BSPs fractions consists of two tapes, linear chain and branched chain. Generally, branched polysaccharides consisting of mannose and glucose are widely recognized as drug carriers in the ratio of $2.4: 1$, because the curved structure is conducive to drug encapsulation. Based on the information, it could be speculated that there probably existed other fractions of BSPs. Thus, the structure of rest fractions remained to be further explored. The two kinds of repeating motifs of BSPs are shown in Figures 2 and 3.

\section{Functional Activities of BSPs}

A book on traditional Chinese medicine Compendium of Materia Medica mentioned: " $B$. striata is astringent and condensation, so it can enter the lung to stop bleeding, muscles and treat sores."

$B$. striata plays a very important clinical role as a traditional Chinese medicine. The main component of $B$. striata is polysaccharides, which plays an irreplaceable role in medical, chemical industry, health care, beauty and moisturization. ${ }^{[43]}$

\section{Hemostatic activity}

Animal experiments showed that the water extract of polar solvent alcohol extract of Bletilla tuber had good hemostasis and coagulation effects on animal organs such as liver, spleen, muscle and blood vessel bleeding. In the study of platelet aggregation and coagulation function in rats, it was found that BSPs exerted hemostatic function, which may activate endogenous and exogenous coagulation system, promote platelet aggregation and achieve hemostasis in rats. ${ }^{[4]}$ The watersoluble part and the alcohol-soluble part of $B$. striata are main effective components of $B$. striata hemostasis, which can significantly improve the maximum platelet aggregation rate and promote platelet aggregation. Guo et al. ${ }^{[45]}$ found that freeze-dried powder absorbed 2-3 times water of its own weight, the degradation rate is positively related to the content of BSPs, and the bacteriostasis is positively related to the 
Table 2 Bletilla striata structure characterization ${ }^{[42]}$

\begin{tabular}{|c|c|c|c|c|c|}
\hline BSPs type & Monosaccharide & Relative molecular weight & Purity/\% & Connection type & Ref. \\
\hline $\mathrm{i}$ & Man : Glc $=3: 1$ & $2.6 \times 10^{5}$ & 98.5 & $\rightarrow 4)$-D-Man- $(1 \rightarrow$ & [35] \\
\hline ii & Man : Glc $=2.4: 1$ & ca. $1.35 \times 10^{5}$ & 95.7 & $\rightarrow 4)-D$-Glc- $(1 \rightarrow$ & {$[1,36]$} \\
\hline iii & - & - & - & $\rightarrow 4)$-D-Man- $(1 \rightarrow$ & [37] \\
\hline iv & Man : Glc $=3.5: 1$ & $0.2 \times 10^{5}$ & 96 & $\begin{array}{l}\rightarrow 6) \text {-D-Glc- }(1 \rightarrow \\
\rightarrow 4)-D \text {-Man- }(1 \rightarrow \\
\rightarrow 4)-D \text {-Glc- }(1 \rightarrow\end{array}$ & [38] \\
\hline $\mathrm{v}$ & - & - & - & - & [33] \\
\hline vi & Man $:$ Glc $=2.8: 1.23$ & $1.68 \times 10^{5}$ & - & $\begin{array}{l}\rightarrow 4) \text {-D-Man- }(1 \rightarrow \\
\rightarrow 4) \text {-D-Glc- }(1 \rightarrow\end{array}$ & [39] \\
\hline vii & Man : Glc $=2.4: 1$ & - & 90 & - & [40] \\
\hline viii & - & $1.383 \times 10^{5}$ & - & - & [41] \\
\hline
\end{tabular}

Abbreviations: Glc, glucose; Man, mannose

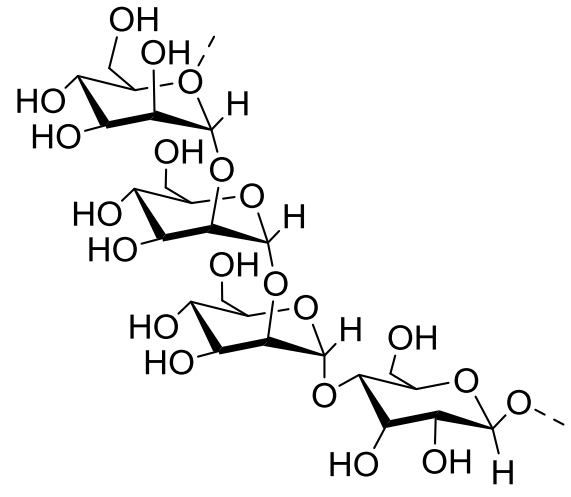

Figure 2 BSPb Man : Glc $=3: 1^{[22]}$

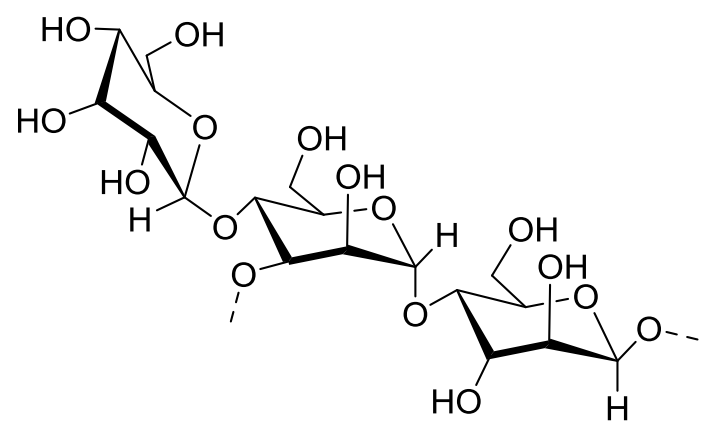

Figure 3 BSP Man: $\mathrm{Glc}=2.4: 1^{[22]}$

content of $\mathrm{N}$-alkylated chitosan. When the ratio of $\mathrm{N}$-alkylated chitosan to BSPs is $4: 1$, the composite freeze-drying powder has the best performance and potential as hemostatic material. $B$. striata can shorten the bleeding time and coagulation time of normal rats and mice. It may be that $B$. striata can make blood cells agglutinate, activate, deform, and aggregate blood platelets. Zhang et al. ${ }^{[46]}$ found that BSPs could promote hematopoiesis in mice with low hematopoietic function. The bioactivity of BSPs is related to its viscosity and solubility. ${ }^{[4]}$ BSPs have promoting effects on hemostasis and coagulation. $^{[48]}$

\section{Anti-oxidant and anti-aging activities}

Anti-oxidant is any substance that can effectively inhibit the oxidation reaction of free radicals when it exists in a low concentration. Its mechanism of action can directly act on free radicals or indirectly consume substances that are prone to free radicals to prevent further reactions. While human body produces free radicals inevitably, it also naturally produces antioxidants that are resistant to free radicals, so as to counteract the oxidative attack of free radicals on human cells. ${ }^{[49]}$

Senescence usually refers to the senescence of cells, which is a short period of near death when cells are subjected to oxidative stress, oncogene activation and DNA damage. ${ }^{[50]}$ Senescence cells can secrete a variety of soluble factors to produce inflammation, accelerate the aging of the body and make the body sick. Some studies showed that promoting the apoptosis of senescence cell can inhibit the senescence of organism. ${ }^{[51]}$

BSPs have anti-aging effects, but their effects cannot be seoarated from the ability of scavenging free radicals and antioxidants. He et al. ${ }^{[52]}$ studied the antioxidant activity of wild BSPs in vitro. They concluded that BSPs had scavenging effects on $\mathrm{O}_{2}{ }^{-}, \cdot \mathrm{OH}$ and DPPH. , and the scavenging capacity was different. Rui et al. ${ }^{[24]}$ conducted antioxidant study of neutral polysaccharides from $B$. striata in vitro. The result showed that BSPs inhibited the production of hydroxyl radicals significantly. Based on the anti-oxidation effect in vitro, the anti-oxidation effect in vivo was studied. The aging model of mice was established by injecting $D$-galactose and the neutral polysaccharides of $B$. striata were given. It can be seen that the neutral polysaccharides of $B$. striata increased the SOD activity of various organs of aging mice induced by $D$-galactose significantly.

\section{Other activities}

BSPs had significant antitumor activities in vivo and in vitro. ${ }^{[2]} \mathrm{Li}$ et al. ${ }^{[53]}$ found that previously investigated the in vivo and in vitro effects of paclitaxel nanoparticle- (PTX-) loaded BSPs on human gastric cancer cells. The results suggested that BSP-loaded paclitaxel nanoparticles could realize enhanced drug delivery and exert an antiproliferative effect on the human gastric gland cancer cell line (MKN45) effectively and safely both in vivo and in vitro. Wu et al. ${ }^{[54]}$ found that BSPs had an obvious anti-stress gastric ulcer effect. Shi et al. ${ }^{[5]}$ found that BSPs have certain immunomodulatory ability on macrophages and can alleviate colitis in mice. In the food field, the polysaccharides of $B$. striata have no toxic side effect and has high application value. Chen et al. ${ }^{[56]}$ pointed out that $B$. striata can be used for brewing wine or stewing soup for medicinal meals, with unique aroma and strong edibility 
Li et al. ${ }^{[57]}$ prepared a compound preservation solution using $B$. striata and applied it on the surface of mango, disclosing that it could effectively delay the weight loss, yellowing and decay of mango, reduce the respiratory strength of mango, inhibit the decline of titrable acid, vitamin $\mathrm{C}$, and soluble total solids content, maintain the good quality of the fruit and extend the storage life of the fruit.

\section{Conclusions}

BSPs are a neutral heteropolysaccharide polymerized by glucose and mannose in a certain proportion. In the past years, a variety of BSPs have been isolated and their diverse structures make the research more challenging. Herein, the structure of BSPs was characterized, which showed that BSPS were a kind of glucomannan. The monosaccharide of BSPs is mainly composed of mannose and glucose, which provides a better scientific basis for further research on BSPs. In addition, BSPs have astringent hemostasis, antioxidant, anti-aging and other active functions. Polysaccharides from $B$. striata have obvious scavenging effects on $\cdot \mathrm{OH}$ and $\mathrm{DPPH} \cdot$, but they have no obvious scavenging effect on $\mathrm{O}_{2}^{-} \cdot$. Polysaccharides can be used as natural food antioxidants. It has low toxicity, high safety and good biocompatibility, and has great potential in medicine and food.

\section{Conflict of Interest}

The authors declare no conflict of interest.

Copyright (c) 2020 Jinyu Yi, Yingying Huang, Chaoyan Zhang. This article is an open access article distributed under the terms and conditions of the Creative Commons Attribution (CC BY) license (http://creativecommons.org/licenses/by/4.0/). The use, distribution or reproduction in other forums is permitted, provided the original author(s) or licensor are credited and that the original publication in this journal is cited, in accordance with accepted academic practice. No use, distribution or reproduction is permitted which does not comply with these terms.

\section{References}

[1] Wang, Y.; Liu, D.; Chen, S. J.; Wang, Y.; Jiang, H. Q.; Yin, H. P. A new glucomannan from Bletilla striata: structural and anti-fibrosis effects. Ftoterapia 2014, 92, 72-78.

[2] Ji, X. L.; Yin, M. S.; Nie, H. A Review of isolation, chemical properties, and bioactivities of polysaccharides from Bletilla striata. Biomed. Res. Int. 2020, 2020, 1-11.

[3] Zhang, Q.; Qi, C.; Wang, H. Biocompatible and degradable Bletilla striata polysaccharide hemostasis sponges constructed from natural medicinal herb Bletilla striata. Carbohydr. Polym. 2019, 226, 115304.

[4] Jiang, F.; Li, M.; Wang, H. Coelonin, an anti-inflammation active component of Bletilla striata and its potential mechanism. Int. J. Mol. Sci. 2019, 20, 4422.

[5] Liu, B.; Zhan, Q.; Wu, X. Effect of Bletilla striata on the prevention of postoperative peritoneal adhesions in abrasion-induced rat model. Evid. Based. Complement. Alternat. Med. 2019, 2019, 9148754.

[6] Shi, Y.; Zhang, B.; Lu, Y. Antiviral activity of phenanthrenes from the medicinal plant Bletilla striata against influenza a virus. BMC Complement. Altern. Med. 2017, 17, 273.

[7] Hung, H. Y.; Wu, T. S. Recent progress on the traditional Chinese medicines that regulate the blood. J. Food Drug Anal. 2016, 24, 221-238.

[8] Ke, C. Y.; Zhao, C. J. Study on the effect of Bletilla striata polysaccharide on ulcerative colitis. J. China Pharm. 2011, 22, 2132-2134 (in Chinese).
[9] Zhao, W. C.; Song, L. J.; Xu, J. H. Application of natural high molecular Bletilla striata polysaccharides in drug preparation. Pharm. Today 2010, 3, 2-3 (in Chinese).

[10] Diao, H.; Li, X.; Chen, J.; Lou, Y.; Chen, X.; Dong, L.; Wang, C.; Zhang, C.; Zhang, J. Bletilla striata polysaccharide stimulates inducible nitric oxide synthase and proinflammatory cytokine expression in macrophages. J. Biosci. Bioeng. 2008, 105, 85-89.

[11] Dong, L.; Xia, S. H.; Luo, Y.; Diao, H. J.; Zhang, J. N.; Chen, J. N.; Zhang, J. F. Targeting delivery oligonucleotide into macrophages by cationic polysaccharide from Bletilla striata successfully inhibited the expression of TNF-alpha. J. Control. Release 2009, 134, 214-220.

[12] He, H. I.; Gu, G. P.; Zhang, W. M. Research on cherry tomato fruits coated with Bletilla gucomannan (Bg) film. Food Sci. 2007, 28, 336-340.

[13] Cheng, Z. Y.; Yang, Y. J.; Liu, Y. Two-steps extraction of essential oil, polysaccharides and biphenyl cyclooctene lignans from schisandra chinensis baill fruits. Northeast Normal University 2014, 96, 162-169 (in Chinese).

[14] Kong, L. S.; Yu, L.; Feng, T. Physicochemical characterization of the polysaccharide from Bletilla striata: effect of drying method. Carbohyd. Polym. 2015, 125, 1-8.

[15] Han, D.; Wang, Y. P.; Bi, Y. J. Extracting method optimization and physical and chemical properties of polysaccharide in Bletilla striata. J. Pharm. Pract. 2013, 31, 35-37.

[16] Qu, Y.; Li, C. X.; Zhang, C.; Zeng, R.; Fu, C. M. Optimization of infrared-assisted extraction of Bletilla striata polysaccharides based on response surface methodology and their antioxidant activities. Carbohydr. Polym. 2016, 148, 345-353.

[17] Jiang, F. S.; Li, W. P.; Huang, Y. F.; Chen, Y. T.; Jin, B.; Chen, N. P.; Ding, Z. S.; Ding, X. H. Antioxidant, antityrosinase and antitumor activity comparison: the potential utilization of fibrous root part of Bletilla striata (Thunb.) Reichb.f. PLoS One 2013, 8, e58004.

[18] Hang-Su, Y. U.; Shi, Z. Z.; Di, L.; Pan, P.; Qian, C. D.; Chen, J. Z.; Ding, Z. S. Comparative study of polysaccharide in fibrous root and tuber of Bletilla striata. J. Yunnan. Univ. Tradit. Chin. Med. 2015, 38, 29-32.

[19] Cai, J. Y.; Liang, Y.; Wu, Q. H.; Hu, J. H.; Liang, H. R.; Wei, K. H. Optimization of extraction of polysaccharide from Bletilla striata and its biological activity. Food Ind. 2018, 39, 45-49.

[20] Kong, J. H.; Shi, J. S.; Sun, D. F. Enzymatic purification of crude polysaccharides extracted from Bletilla striata roots. Food Sci. 2009, 30, 52-56.

[21] Jin, S. Progress on extraction of polysaccharide from Bletilla striata and its application in food. Process. Technol. 2018, 1, 124-127.

[22] Chen, Z. Y.; Cheng, L. Z.; He, Y. C. Extraction, characterization, utilization as wound dressing and drug delivery of Bletilla striata polysaccharide: A review. Int. J. Biol. Macromol. 2018, 120, 2076-2085.

[23] Chen, J. Y.; Zhang, W. M.; Wang, X. D. A comparative study on the sulfation and biological activity of Bletilla striata polysaccharide. Food Sci. 2007, 28, 27-31 (in Chinese).

[24] Rui, H. Y.; Wu, G. R.; Chen, J. Y. Experimental study on the antioxidative effect of Bletilla striata neutral polysaccharide. Journal of Nanjing Normal University 2003, 26, 94-98 (in Chinese).

[25] Wang, B.; Xu, S.; Huang, L. J. Separation, purification and structure study of Bletilla striata polysaccharide BSPI-A. Food Sci. 2010, 31, 120 (in Chinese).

[26] Zhang, Y. A comparative study on the extraction methods of poria cocos polysaccharides. Chin. J. Pract. Med. 2012, 7, 249 (in Chinese).

[27] Lu, J. Q.; Zhang, Y. D. Experimental study of Bletilla striata. Chin. Pat. Med. 1996, 18, 2-3 (in Chinese).

[28] Liu, F. Q.; Wang, Y. P.; Han, D. Extraction of Bletilla striata polysaccharide and its relative molecular mass determination and structure study. Chin. Pat. Med. 2013, 35, 2291-2293 (in Chinese). 
[29] Peng, Q.; Li, M.; Xue, F. Structure and immunobiological activity of a new polysaccharide from Bletilla striata. Carbohyd. Polym. 2014, 107, 119-123.

[30] Tomoda, M.; Matsumoto, K.; Shimizu, N.; Gonda, R.; Ohara, N. Characterization of a neutral and an acidic polysaccharide having immunological activities from the root of paeonia lactiflora. Biol. Pharm. Bull. 1993, 16, 1207.

[31] Wang, C. M.; Sun, J. T.; Yi, L. Y.; Xue, W. H.; Diao, H. J.; Lei, D.; Chen, J. N.; Zhang, J. F. A polysaccharide isolated from the medicinal herb Bletilla striata induces endothelial cells proliferation and vascular endothelial growth factor expression in vitro. Biotechnol. Lett. 2006, 28, 539-543.

[32] Wang, B.; Sha, X. U.; Huang, L. J.; Wang, Z. F. Isolation, Purification and structural characterization of a polysaccharide fraction from stem tuber of Bletilla striata, named BSPI-A. Food Sci. 2010, 31, 120-123.

[33] Zhang, Q.; Wan, Y.; Zhao, W. C. Preparation, characterization and in vitro antitumor effect of cholesterol succinyl Bletilla striata polysaccharide-loaded paclitaxel nanoparticles. Biomed. Res. 2017, 21, 9638-9646.

[34] Wang, Y.; Liu, J. J.; Li, Q.; Wang, Y. T.; Wang, C. M. Two natural glucomannan polymers, from konjac and Bletilla, as bioactive materials for pharmaceutical applications. Biotechnol. Lett. 2015, $37,1-8$.

[35] Guan, Q. X.; Sun, D. D.; Zhang, G. Y. Docetaxel-loaded selfassembly stearic acid-modified Bletilla striata polysaccharide micelles and their anticancer effect: preparation, characterization, cellular uptake and in vitro evaluation. Molecules 2016, 21, 2-14.

[36] He, X. R.; Wang, X. X.; Fang, J. C. Bletilla striata: medicinal uses, phytochemistry and pharmacological activities. J. Ethnopharmacol. 2017, 195, 20-38.

[37] Guan, Q. X.; Zhang, G. Y.; Sun, D. D. In vitro and in vivo evaluation of docetaxel-loaded stearic acid-modified Bletilla striata polysaccharide copymer micelles. PLoS One 2017, 12, 1-15.

[38] Zhang, M. S.; Sun, L.; Zhao, W. C. Cholesteryl-modification of a glucomannan from Bletilla striata and its hydrogel properties. Molecules 2014, 19, 9089-9100.

[39] Zhu, J. X.; Guo, X. X.; Guo, T. T. Novel pH-responsive and self-assembled nanoparticles based on Bletilla striata polysaccharide: preparation and characterization. RSC Adv. 2018, 8, 40308-40320.

[40] Dang, T. T.; Wang, J.; Tang, P. Preparation and characterization of reactive white oxygen and polysaccharide-loaded micelles in response to active oxygen. China Tradit. Herb Drugs 2018, 49, 5548-5553 (in Chinese).

[41] Zhan, X. D.; Jia, L. X.; Niu, Y. M. Targeted depletion of tumour-associated macrophages by an alendronate-glucomannan conjugate for cancer immunotherapy. Biomaterials 2014, 35, 10046-10057.

[42] Guo, T. T.; Zhu J. X.; Yang Y. Molecular design and application progress of Bletilla striata polysaccharides as nanometer drug delivery systems. Chin. J. Pharm. 2019, 50, 9 (in Chinese).

[43] Sun, L. L.; Yang, Y. H.; Liu, K. H. Research on Bletilla striata materia medica. China Tradit. Herb. Drugs 2010, 12, 1965-1968 (in Chinese).

[44] Dong, L.; Dong, Y. X.; Liu, X. X. Effects of Bletilla striata polysaccharide on platelet aggregation, coagulation function and expression of TXB2 and 6-keto-PGF1 $\alpha$ in rats. J. Guiyang Coll. Tradit. Chin. Med. 2014, 39, 459-462 (in Chinese).

[45] Guo, L. L. Preparation of $N$-alkylated chitosan/Bletilla striata polysaccharide composites. Appl. Chem. Ind. 2020, 3, 632-637 (in Chinese).

[46] Zhang, Y.; Zhou, Q. X.; Lai, S. Effect of Bletilla striata polysaccharide on bone marrow hematopoietic cells. Pharmacol. Clin. Chin. Mater. Clin. Med. 2009, 25, 35 (in Chinese)

[47] Luo, L.; Yang, F.; Wang, Y.; Zhang, Juan. Experimental and applied research of Bletilla striata polysaccharides. Hubei J. Tradit. Chin. Med. 2016, 38, 69-72 (in Chinese).

[48] Wu, G. j.; Liu, H. I.; Wang, H. Experimental study on the effect of Bletilla striata polysaccharides on normal mice's bleeding and coagulation time. Heilongjiang J. Tradit. Chin. Med. 2011, 3, 49-50 (in Chinese).

[49] Kong, L. S.; Molecular weight, hygroscopicity and moisturizing performance of polysaccharide from Bletilla striata. Daily Chem. Ind. 2015, 2, 94-98 (in Chinese).

[50] Kang, X. P.; Liang, C.; Jin, G. Q.; Zhang, X. L. Effects of bushen recipe on nerve cell apoptosis and learning and memory in aging rats induced by $D$-galactose. Pharmacol. Clin. Chin. Mater. Clin. Med. 2014, 30, 8-11 (in Chinese).

[51] Childs, B. G.; Baker, D. J.; Kirkland, J. L. Senescence and apoptosis: dueling or complementary cell fates. Embo. Rep. 2015 , 15, 1139-1153.

[52] He, X. M.; Gu, F. L.; Huang, R. Z.; Han, B. X.; Chen, N. F. Optimization of ultrasonic-assisted extraction of Bletilla striata polysaccharides and in vitro antioxidant activity. J. West Anhui Univ. 2017, 33, 1-5 (in Chinese).

[53] Li, X. C.; Bai, G. In vivo and in vitro effects of Bletilla striata polysaccharide-loaded paclitaxel nanoparticles on human gastric cancer cells. Trop. J. Pharm. Res. 2019, 18, 13-17.

[54] Wu, G. J.; Su, X. Y.; Xia, X. L. Experimental observation of the effect of Bletilla striata polysaccharide on stress gastric ulcer in rats. Inf. Tradit. Chin. Med. 2011, 28, 43-45 (in Chinese).

[55] Shi, S.; Luo, H. Z.; Luo, Y. Therapeutic effect of Bletilla striata polysaccharide on ulcerative colitis in mice. J. China Med. Univ. 2012, 43, 535-540 (in Chinese)

[56] Shang, J. Research progress on extraction technology of Bletilla striata polysaccharide and its application in food. Modern Food 2018, 124-127 (in Chinese).

[57] Li, X. L.; Wang, Q.; Zhao, B. Study on the effect of compound preservative of Bletilla striata on mango. J. Anhui Agric. 2011, 39, 7726-7728 (in Chinese)

Received May 22, 2020 Accepted August 31, 2020 